\title{
Kernos
}

Revue internationale et pluridisciplinaire de religion grecque antique

$24 \mid 2011$

Varia

\section{Le sferette bronzee iscritte da Himera}

\section{Antonietta Brugnone}

Edizione digitale

URL: http://journals.openedition.org/kernos/1936

DOI: 10.4000/kernos.1936

ISSN: 2034-7871

\section{Editore}

Centre international d'étude de la religion grecque antique

Edizione cartacea

Data di pubblicazione: 1 gennaio 2011

ISSN: 0776-3824

Notizia bibliografica digitale

Antonietta Brugnone, "Le sferette bronzee iscritte da Himera », Kernos [En ligne], 24 | 2011, mis en ligne le 01 février 2014, consulté le 03 mai 2019. URL : http://journals.openedition.org/kernos/1936 ; DOI : 10.4000/kernos. 1936 
Kernos 24 (2011), p. 77-94.

\title{
Le sferette bronzee iscritte da Himera
}

\begin{abstract}
The focus of the study is $(\mathbb{1} 1)$ the analysis of an inscribed bronze spherule discovered in the temenos on the Piano di Imera. The document belongs to a small group of similar inscriptions, consisting of three bronze spherules inscribed with a sample formulaic structure: the divinity's name. Firstly, the article aims at providing a new framework of interpretation for the word ė $\pi \lambda \nu \sigma \alpha \mu \varepsilon^{\prime}(v) \alpha \varsigma$ as Eileithyia's epithet, the Greek deity of birth to whom literary evidence attributes the power of 'losing up', 'freeing'. Secondly we analyze other bronze spherules from the Piano di Imera ( $(2)$. This method allows to examine and compare various kind of evidence in order to get a full understanding of the particular use of these little objects, i.e. their role in cleromancy rituals (\$ 3).
\end{abstract}

Résumé : L'étude est centrée sur l'analyse d'une petite sphère en bronze inscrite que l'on a trouvée à l'intérieur du temenos dans le Piano di Imera ( $(1)$. Elle appartient au même groupe de documents dont trois exemplaires étaient connus avec les noms des divinités au nominatif ou au génitif. Dans la première partie de cette étude est examiné le texte de l'inscription afin d'évaluer l'hypothèse qu'il s'agit d'une épithète d'Ilithye, la déesse grecque des naissances à qui les sources anciennes attribuent la fonction de " dénouer », de "libérer », d'« affranchir »; dans la deuxième partie est reprise l'analyse des petites sphères déjà publiées $\left(\mathbb{\int} 2\right)$, mais aussi de plusieurs types de témoignages pouvant mettre en lumière l'usage de ces petits objets dans les rituels divinatoires $(\$ 3)$.

Tra i materiali rinvenuti nel temenos sul Piano di Imera figura una sferetta bronzea iscritta, proveniente da scavi clandestini, che per il peso, la forma, il colore, i caratteri epigrafici e il testo, appare riconducibile allo stesso gruppo di documenti di cui erano già noti tre esemplari ${ }^{1}$. Per questi documenti non ho trovato confronti in ambito santuariale, se si escludono la sferetta anepigrafe proveniente dall'Heraion di Capo Lacinio, da un contesto datato alla seconda metà del VI sec. a.C. ${ }^{2}$ e quella su cui figura il segno E, rinvenuta nell'Asklepieion di Agrigento ${ }^{3}$. Sferette anepigrafi in bronzo fuso sono state trovate «in numerosi esemplari per corredo» nella necropoli di Locri Epizefirii e sono state interpretate come testimonianze di giochi di fanciulle ${ }^{4}$.

${ }^{1}$ Manni Piraino (1974), p. 267-269, n. 4, tav. XLIII, fig. a-b; EAD. (1984-85), p. 253-254, n. 2-3, tav. XX, 2-2b.

2 SPADEA (1996), p. 113, n. 27.

${ }^{3}$ De Miro (2003), p. 181, n. 275, fig. 75, tav. LXXXVIII, 2.

${ }^{4}$ Cerchial (1982), p. 291. 


\section{La sferetta inedita}

La sferetta inedita, conservata nell'Antiquarium di Himera (H82.51), è leggermente schiacciata ed è ricoperta da una patina verde cupo ben conservata. Diam. cm 2,1; peso gr. 43,69. L'iscrizione è incisa a freddo e sulla base delle caratteristiche paleografiche è databile all'ultimo quarto del V sec. a.C. Alt. lett. cm 0,6-0,4. Epsilon con i tratti paralleli orizzontali; $p i$ con il terzo tratto relativamente lungo; lambda con l'angolo in alto; sigma a quattro tratti; alpha con la sbarra appena discendente verso sinistra; il my ha la forma di un doppio lambda (Fig. 1) ${ }^{5}$.

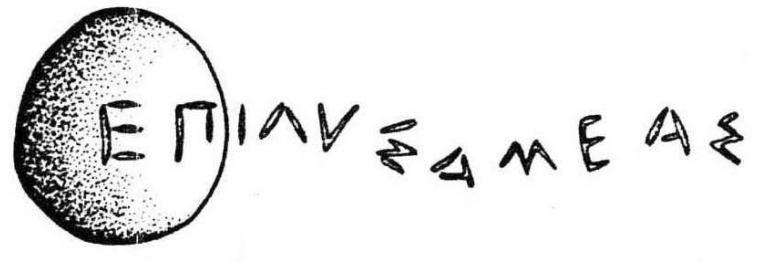

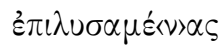

Fig. 1

Se ipotizziamo che l'incisore abbia omesso un ny possiamo leggere il genitivo femminile del participio aoristo del verbo é $\pi \imath \hat{u} \varepsilon \iota \nu$, usato al medio con il significato di 'sciogliere', liberare', 'manomettere', ecc. La conservazione del suono a nella desinenza del genitivo potrebbe essere ascritta alla componente dorica della popolazione di Himera che, presente già al momento della fondazione, si sarebbe notevolmente rafforzata dopo la cacciata di Terillo e, in particolare, nel 476 a.C., quando Terone integrò nel corpo civico imerese nuovi coloni di origine dorica ${ }^{6}$. Dalla forma maschile del participio deriva l'antroponimo 'E $\iota \lambda \cup \sigma \alpha \mu \varepsilon v o ́ s$ che figura in un'epigrafe ateniese della fine del $\mathrm{V}$ sec. a.C.7; il

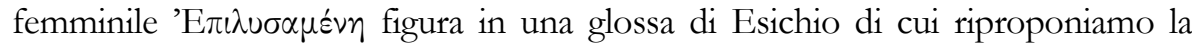

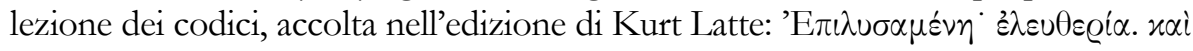

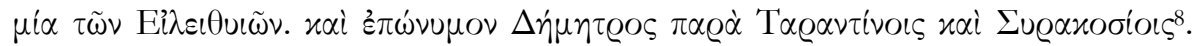
La glossa lascerebbe ipotizzare che nella nuova sferetta figuri un teonimo o un'epiclesi di Demetra. Nelle precedenti edizioni ${ }^{9}$ la glossa era stata considerata un erroneo duplicato di un'altra glossa, 'E $\lambda \varepsilon v \theta \omega^{\cdot}$ 'éexo $\mu \alpha$. Eì $(\varepsilon) i \theta v \iota \alpha$ che era stata

5 A Himera il lambda con l'angolo in alto e il sigma a quattro tratti cominciano a sostituire rispettivamente il lambda 'calcidese' e il sigma a tre tratti nel secondo quarto del V sec. a.C. (BRUGNONE 1995, p. 1303-1307).

6 Diodoro, XI, 49, 3.

7 IG I3, 1190 ; II, 131. BECHTEL (1917, p. 569) seppure dubitativamente, fa derivare l'antroponimo dall'epiclesi di Demetra.

${ }^{8}$ LATTE (1966), II, p. 162.

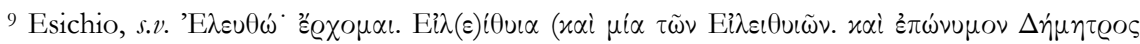

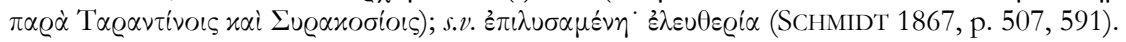




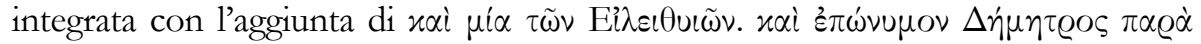

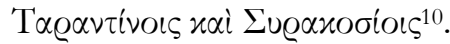

In Sicilia non sono note altre testimonianze epigrafiche della divinità greca delle nascite, se si esclude un vaso a vernice nera del Museo di Agrigento su cui figura il graffito: $\tau \tilde{\alpha} \varsigma^{\prime} \mathrm{E} \lambda \varepsilon v \theta i \alpha \varsigma^{11}$.

Il nome della divinità delle nascite - di cui si conoscono numerose varianti ${ }^{12}$ è stato ricondotto da alcuni linguisti allo strato linguistico pre-greco ${ }^{13}$; altri hanno

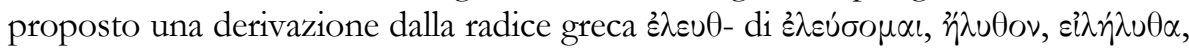
che darebbe al teonimo il significato di 'colei che viene' o 'che fa venire'14, oppure hanno ipotizzato che un nome non greco sia stato accostato alla stessa radice, per etimologia popolare ${ }^{15}$. Secondo un'altra ipotesi il teonimo sarebbe riconducibile alla radice di *ย่ $\lambda \varepsilon u \theta \varepsilon \tilde{\nu} \nu$, 'essere libero' o 'rendere libero'; dalla stessa radice deriverebbe é $\lambda \varepsilon \dot{\theta} \theta \varepsilon \varrho o s$, lat. liber ${ }^{16}$.

Senza entrare nel merito di una disputa di carattere linguistico che esula dalle mie competenze, vorrei cercare di capire se le prerogative e le funzioni di Ei $\lambda \varepsilon^{i} \theta v \iota \alpha$, che possiamo ricostruire attraverso la lettura delle fonti, siano coerenti con l'epiteto 'E $\pi \iota \nu \sigma \sigma \alpha \mu \varepsilon ́ v \eta$.

Nell'Iliade il teonimo compare ora al singolare, Ei $\lambda \varepsilon^{i} \theta \nu \iota \alpha$, ora al plurale

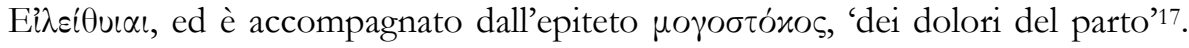

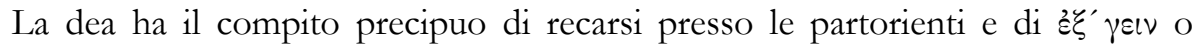

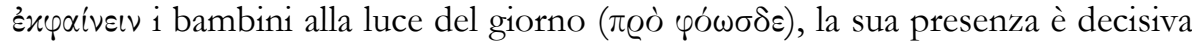
perché il parto si compia ${ }^{18}$. E infatti, nell'inno omerico ad Apollo, Era, volendo impedire la nascita del dio, trattiene Ilitia sulla cima dell'Olimpo finché Iride, eludendo la sorveglianza della madre, la convince a seguirla fino a Delo dove Leto già da nove giorni e nove notti era trafitta dalle doglie ${ }^{19}$ : «solo quando Ilitia

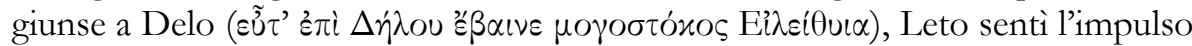

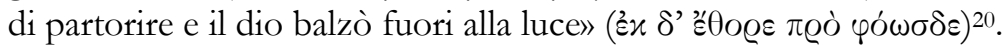
159 .

${ }^{10}$ Cf. O. JESSEN, s.v. Eleutho, RE V 2 (1905), col. 2356; ID., s.v. Epilysameme, RE VI (1907), col.

11 Dubois (1989), p. 205, n. 67; Manganaro (1992), p. 208, fig. 1; (1997), p. 75.

12 O. JESSEN, s.v. Eileithyia, RE V 2 (1905), col. 2102.

13 WaCKernagel, apud NiLSSON (1950), p. 518-523.

14 Schulze (1892), p. 259-263. Anche Heubeck (1972), p. 95, propende per questa interpretazione. Vd. pure Chantraine (1968), s.v. Ei $\lambda \varepsilon i \theta u \iota \alpha$; Pingiatoglou (1981), p. 11-12.

${ }^{15}$ Cf. Puhvel (1964), p. 165.

${ }^{16}$ Cf. Puhvel (1964), p. 165; Gérard-Rousseau (1968), p. 102. Heubeck (1972, p. 95)

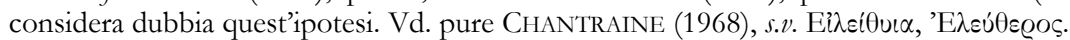

17 Omero, Iliade XI, 269-271.

18 Omero, Iliade XVI, 187-188; XIX, 103-104, 118-119. Cf. JESSEN, s.v. Eileithyia, l.c. (n. 12), col. 2102-2103; Pingiatoglou (1981), p. 13-14; R. Olmos, s.v. Eileitbyia, LIMC III.1 (1986), p. 686.

${ }^{19}$ Inni omerici III, 91-92.

${ }^{20}$ Inni omerici III, 115-119. 
Forme verbali che derivano dalla radice $\varepsilon \lambda \varepsilon v \theta-$-, 'venire', figurano, riferite alla

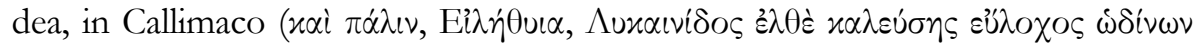

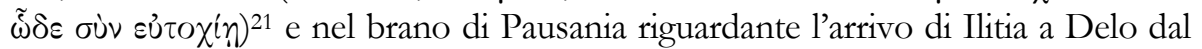

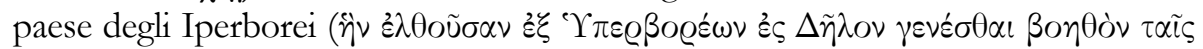
$\Lambda \eta \tau O \tilde{u} \varsigma \hat{\omega} \delta \tilde{\imath} \sigma)^{22}$.

Si deve arrivare alla lessicografia bizantina per trovare esplicitata la derivazione

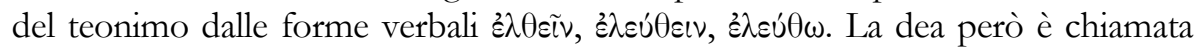
Ei $\lambda \varepsilon^{\prime} \theta u \iota \alpha$ non, come ci si potrebbe aspettare, per il fatto che "viene" in aiuto delle partorienti, ma per il fatto che i bambini "vengono alla luce" grazie al suo

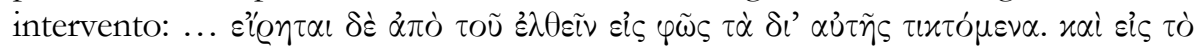

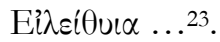

In altri autori la funzione costitutiva della personalità di Ilitia è quella di

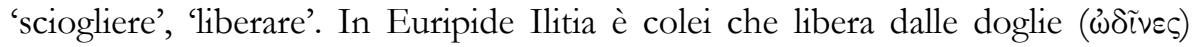
Auge, la figlia di Aleo, quando, violentata da Eracle, dà alla luce Telefo: ...

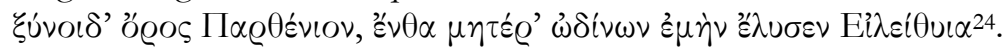

Negli Idilli di Teocrito Ilitia è la dea $\lambda \cup \sigma i \zeta \omega v o \varsigma$ 'che scioglie la cintura'25. In

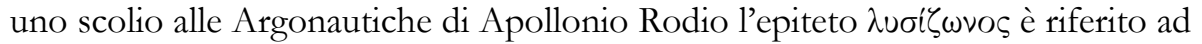
Artemide e spiegato con il fatto che le donne che partoriscono per la prima

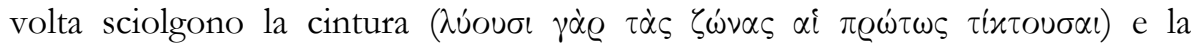
consacrano alla dea ${ }^{26}$. Nella letteratura orfica Prothyraia, invocata anche come Ilitia e Artemide Ilitia, è la dea $\lambda \cup \sigma i \zeta \omega \nu o \varsigma$ che 'scioglie i dolori nelle terribili

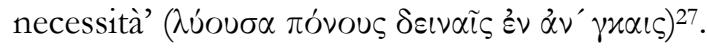

In altre tradizioni invece $i$ nodi che impediscono il parto e che Ilizia deve sciogliere non hanno alcun rapporto con la figura della partoriente, ma, come nella magia simpatica, sono estranei ad essa: sono le mani intrecciate, le gambe accavallate, le braccia strette intorno ai ginocchi delle Moirai e di Ilitia/Lucina ${ }^{28}$.

21 Callimaco, Epigrammi, LIII.

22 Pausania, I, 18, 5. Vd. Olmos, s.v. Eileithyia, l.c. (n. 18), p. 685.

23 Etym. Gud. b 277, 20-22; e 415, 5-7. Vd. pure Eustazio, Comm. ad Hom. Il. III 195, 1-2; III, 196, 8-9; Eustazio, Comm. ad Hom. Od., II, 198, 21; Scholia in Oppianum, 1, 477, 9-11.

24 Euripide, fr. 696 (ed. NAuCK). Vd. Ch. BAuChHenss-Thüriede, s.v. Auge, LIMC III 1 (1986), p. 45; Olmos, s.v. Eileithyia, cit. (n. 22), p. 686. Il concetto di liberare dalle doglie figura anche in un epigramma dell' Anthologia Palatina (VI, 273 ed. BECKBY).

25 Teocrito, Idilli, 17, 60-63. Cf. Jessen, s.v. Eileithyia, cit. (n. 18), col. 2104. Secondo Plinio (Storia naturale XXVIII, 9) la cintura che deve essere sciolta per favorire il parto non è quella della partoriente, ma quella del padre del nascituro che deve slacciarla, legarla intorno alla donna e di nuovo slacciarla.

26 Schol. in Ap. Rh., Arg. XXXIII, 13-14.

27 Inni Orfici II, 7-11. In Ovidio (Fasti III, 256-258) invece la donna gravida per accelerare il parto non scioglie la cintura, ma i capelli.

28 Antonino Liberale, Metamorfosi I, 29, 2-3; Ovidio, Metamorfosi IX, 295-304. Vd. pure Plinio, Storia naturale XXVIII, 17. 
Le funzioni alle quali rimandano le interpretazioni proposte dai linguisti sono associate in due brani di autori tardi nei quali Ilitia è la dea 'che viene', perché solo dopo il suo arrivo il parto può compiersi, ed è anche la dea che scioglie i legami dell'utero. Nel de natura deorum di Anneo Cornuto le partorienti invocano Ilitia, la dea che gira senza posa e si muove intorno alla terra, perché vada da loro benigna e $\lambda$ ui $\zeta \omega \nu o \varsigma$ e sciolga quello che serra l'utero, in modo che il concepito cada fuori più agevolmente e con minore sofferenza ${ }^{29}$. Nonno, nel riportare la tradizione della nascita di Beroe da Cipride, unisce l'immagine di Ilitia 'che viene' e quella di Ilitia 'che scioglie', ma invece di $\lambda \cup \sigma i \zeta \omega \nu o \varsigma$, usa il

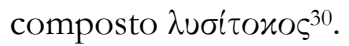

Da questa rassegna, seppure cursoria e parziale, delle fonti relative ad Ilitia si desume che l'epiteto Epilysamene, colei 'che scioglie', è assolutamente coerente con una parte significativa delle tradizioni riguardanti la sfera d'azione della dea delle nascite. Quanto alla notizia dell'attribuzione dello stesso epiteto a Demetra presso i Tarantini e i Siracusani, mi limito a ricordare in questa sede che le dediche rinvenute a Heraclea di Lucania ci restituiscono l'immagine di un'altra Demetra preposta allo scioglimento dei legami - che non sono i legami della nascita, ma i legami della schiavitù - attraverso forme sacrali di manomissione ${ }^{31}$.

\section{Le altre sferette}

Le altre sferette iscritte da Himera, di cui recentemente Marina Sclafani ${ }^{32}$ ha sottolineato l'appartenenza alla «medesima classe di materiali», erano state pubblicate da Maria Teresa Manni Piraino: la prima era stata rinvenuta nell'ambiente 2 della stoa ovest del temenos sul Piano di Imera, nel terreno agricolo, le rimanenti provenivano da un sequestro.

${ }^{29}$ Cornuto, Sulla natura degli dei, 73, 7-12.

30 Nonno, Dionisiache XLI, 158-167: Quando Cipride giunse al termine della gestazione, «preve-

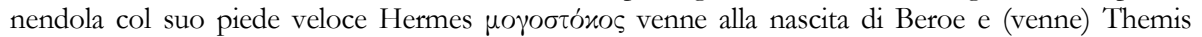

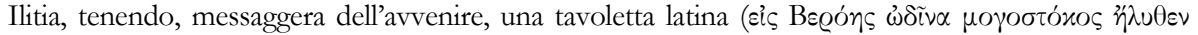

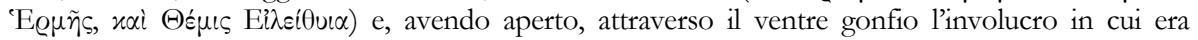
chiuso il bambino, rendeva leggero il dardo acuto di un parto che era arrivato a maturazione, tenendo in mano le leggi di Solone: appoggiando la schiena appesantita alla dea che presiede alle

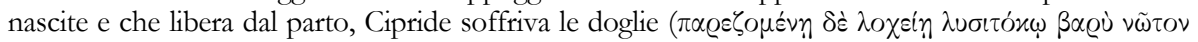

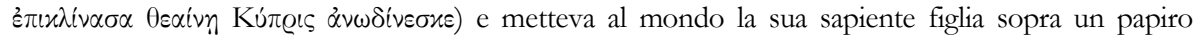
attico, come le donne spartane mettevano al mondo i lori figli su uno scudo rotondo di cuoio. La traduzione segue quella di CHUVIN, FAYANT, Paris, 2006, con una sola eccezione: «... Hermès tenant une tablette en latin, messagère de l'avenir, vint à la naissance de Béroé, pour en apaiser les douleurs. Et c'est Justice qui fut Eileithyia (l'Accoucheuse) ...».

31 Maddoli (1986), p. 99-107. Cf. SARTORi (1992), p. 269-277; Lippolis, NAFissi, GarrafFo (1995), p. 179-180. A Crotone invece tale funzione era attribuita a Hera: GiANGIULIO 1982, p. 1926; MADDOLI (1987-88), p. 141-156; GiANGiUlio (1989), p. 58 ss.

32 SCLAFANi (2007), p. 247-265. 
2.1. H74.19. Forma sferica leggermente schiacciata. Bronzo con patina verde cupo ben conservata. Diam. cm. 2,2-2; peso gr. 47,2. Alt. lett. cm 0,5-0,7. Delta a triangolo isoscele col tratto sinistro staccato dagli altri due; sigma a quattro tratti; l'o lungo è espresso con $\mathrm{O}$; l'e lungo con $\mathrm{H}$. Iscrizione incisa a freddo lungo la circonferenza maggiore, databile, sulla base delle caratteristiche paleografiche, all'ultimo quarto del V sec. a.C. (Fig. 2) ${ }^{33}$.

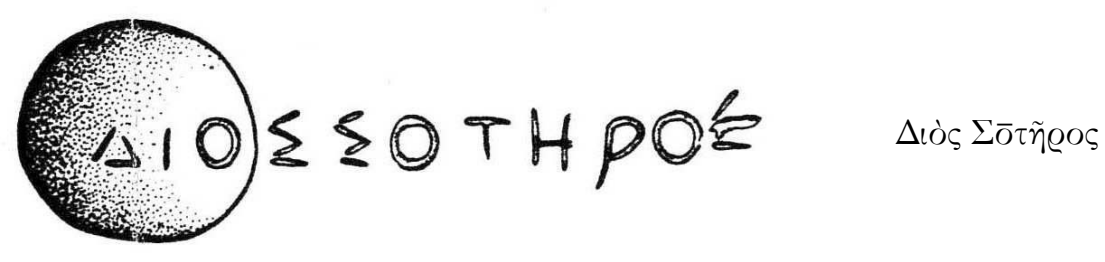

Fig. 2

L'editrice pensava che si trattasse di una «glans missile con acclamazione a divinità», senza escludere però l'ipotesi dell'«offerta votiva» ${ }^{34}$. L'epiklesis ricorre, accanto alla figura della Ninfa sacrificante, in una serie di didrammi di Himera, variamente datati tra il 472 e il 409 a.C. ${ }^{35}$.

Le altre due sferette che per forma, materiale e caratteristiche paleografiche, sono simili alla prima, sulla base delle dichiarazioni della persona che le deteneva al momento del sequestro, sarebbero state trovate in località Piano Lungo, sul versante est del Piano di Imera, nella proprietà Pirrone, in un'area non lontana dal luogo in cui era stato trovato l'esemplare col nome di Zeus Soter. Il lambda con lo spigolo in alto, il delta a triangolo isoscele, il sigma a quattro tratti e il segno $\mathrm{H}$ usato per esprimere il suono è confermano l'adozione da parte degli Imeresi dell'alfabeto ionico ${ }^{36}$.

2.2. HA 1824. Forma sferica leggermente schiacciata. Bronzo con patina verde cupo ben conservata. Diam. cm. 2,1-2; peso gr. 41,96. Iscrizione incisa a freddo, databile all'ultimo quarto del V sec. a.C. Alt. lett. cm. 0,5-0,837.

33 Manni Piraino (1974), p. 267-269, n. 4, tav. XLIII, fig. 6 a-b; MAnni Piraino (1976-77), p. 279; Dubois 1989, p. 14-15, n. 13; SClafani (2007), p. 247 ss. Vd. pure BonaCaSA (1982), p. 56; MANGANARO (1995), p. 104; TORELli (2003), p. 677.

34 Cf. Bonacasa (1980), p. 46. Nella XII Olimpica di Pindaro, per Ergotele di Himera, si invoca Zeus Eleutherios, padre di Tyche Soteira (Pindaro, Olimpiche, XII, 1-3). Sulle testimonianze del culto di Zeus Soter in Sicilia, vd. MANGanaro (1977), p. 153, 156, n. 43; ID. (1996), p. 80; ID. (2003), p. 5-8; GROTTA (2009), p. 375-377, in part. nota 2, p. 375.

35 Franke, Hirmer (1972), p. 44, n. 66, tav. 20: tra il 472 ed il 450 a.C.; Tusa Cutroni (1972), p. 120, n. 6: 450-430 a.C.; Mildenberg, HurTer (1985), n. 617, p. 42: intorno al 430 a.C. Vd. pure CaCCAMO CALTABiano (1990), p. 424-425.

36 Vd. supra, n. 5.

37 MAnni PiRAino (1984-85), p. 254, n. 2, tav. XX, 2-2b; SEG 36, 834a; SCLAfAni (2007), p. 248-250, fig. 3; Dubois (2008), p. 36, n. 17, 2. Vd. pure Manganaro (1995), p. 104; Miranda (1998), p. 231. 


\section{${ }^{\top} \mathrm{H} \varrho \alpha \lambda$ غ́os}

Non mi soffermerò sulla ben nota tradizione diodorea riguardante le sorgenti calde che le Ninfe avevano fatto sgorgare nel territorio imerese, all'arrivo di Eracle ${ }^{38}$. La posizione di primo piano dell'eroe nell'orizzonte cultuale di Himera ha trovato significative conferme nella documentazione epigrafica ${ }^{39}$ e archeologica ${ }^{40}$.

2.3. HA 1825. Forma sferica leggermente schiacciata. Bronzo con patina verde cupo ben conservata. Diam. cm. 2,1; peso gr. 40,97. Iscrizione incisa a freddo, databile all'ultimo quarto del V sec. a.C. Alt. lett. cm. 0,7-0,541.

\section{$\Lambda \varepsilon \cup \varkappa \alpha \theta \varepsilon ́ \alpha$}

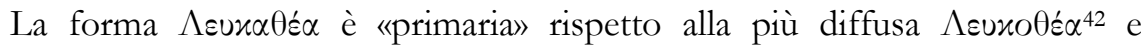
nell'area tirrenica è già nota a Neapolis dall'epitaffio di età tardo-repubblicana di una sacerdotessa della dea ${ }^{43}$.

La connessione di Leucothea con la sfera della navigazione compare già nella tradizione omerica che identifica la dea con Ino, figlia di Cadmo re di Tebe. Un tempo mortale, Ino aveva avuto l'onore di diventare una dea, la dea bianca, protettrice della navigazione. Tramutatasi nell'uccello marino chiamato aithuia, Leucothea salva Odisseo quando, giunto in vista del paese dei Feaci, sta per soccombere, colpito dalla collera di Poseidone. Sorgendo dalle onde la dea porge

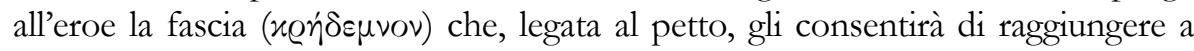
nuoto la terra dei Feaci ${ }^{44}$. Ino si sarebbe gettata in mare dalla rupe Moluride, lungo la strada tra Megara e Corinto, con Learco e Melicerte, i figli avuti da Atamante ${ }^{45}$ o solo con Melicerte, il più giovane ${ }^{46}$. Il salto in mare, katapontismos, è alla base dell'eroizzazione di Ino, accolta tra le Nereidi col nome di Leucothea ${ }^{47}$, e di Melicerte, diventato Palemone, a cui sarebbero stati dedicati i giochi istmici ${ }^{48}$.

38 Timeo, 566 F 165 (ed. JACOBY); Diodoro, IV 23, 1; V 3, 4.

39 Manni Piraino (1970), p. 345-346, n. 2-3, fig. 18-19, tav. LXXIX, 5, 7.

40 Sulle testimonianze archeologiche del culto di Eracle a Himera, vd. BOnACASA (1980), p. 45-49; ID. (1991), p. 1431-1439; ID. (1992), p. 146-150; ID. (2005), p. 241-253, in part. p. 249253; CONSOLI (2008), p. 58-70.

41 Manni Piraino (1984-85), p. 254, n. 3; SEG 36, 834b; SCLAfani (2007), p. 248-250, fig. 3; Dubois (2008), p. 36, n. 17, 2. Vd. pure MANGanaro (1995), p. 104; Miranda (1998), p. 231.

42 WaCKernagel (1925), p. 44-46. Cf. Giangiulio (1986), p. 114.

${ }^{43}$ Miranda (1995), p. 28-30, n. 94.

${ }^{44}$ Omero, Odissea V, 333-353, 458-462. Cf. Breglia Pulci Doria (1998), p. 99-101.

45 Euripide, Medea, 1286 ss.

46 Pausania, I, 44, 7-8; Apollodoro, Biblioteca I, 9, 1-2, 80-84; III, 4, 2-3, $26-29$.

47 Pausania, I, 42, 7.

48 Pausania, I, 44, 8; II 1, 3. 
Un'altra tradizione, riportata da Diodoro ${ }^{49}$, identifica Leucothea con Halia, la sorella dei Telchini - gli antichi abitanti di Rodi - amata da Poseidone; i figli di Posidone e Halia, resi pazzi da Afrodite, si sarebbero uniti alla madre e questa, gettatasi in mare, sarebbe stata chiamata Leucothea.

La carta di distribuzione del culto nell'area tirrenica mostra che erano consacrati alla dea gli approdi e i punti di riferimento più importanti per le navi che dalla Sicilia occidentale o dallo stretto di Messina si dirigevano verso la Campania e da qui, costeggiando il Lazio e l'Etruria, procedevano verso Nord fino a Massalia ${ }^{50}$. Il culto di Leucothea è attestato infatti a Massalia ${ }^{51}$, Elea ${ }^{52}$ e nelle città etrusche e latine: la Mater Matuta del santuario emporico dell'area di Sant'Omobono, a Roma, era stata interpretata come Leucothea ${ }^{53}$, la dea etrusca del santuario di Pirgi ora come Lencothea ${ }^{54}$ ora come Ilitia $a^{55}$. In un'altra località del Lazio, Tivoli, la ninfa Albunea (dal lat. albus 'bianco') ${ }^{56}$, stando alla testimonianza di Servio ${ }^{57}$, era chiamata da alcuni Leucothea. Il teonimo ricorre anche nella toponomastica del Tirreno meridionale: a sud di Capri incontriamo un'isoletta chiamata Lencothea non localizzabile con precisione ${ }^{58}$; uscendo dal golfo di Posidonia, di fronte a Punta Licosa, la Sirena $\Lambda$ surwoía dà il nome a un'isoletta che alcuni vorrebbero identificare con quella chiamata Leucothea ${ }^{59}$.

Il rinvenimento imerese sembra confermare l'ipotesi del carattere originariamente euboico del culto neapolitano. Tale ipotesi si fonda sul fatto che la zona dell'Asia Minore in cui si addensano le testimonianze della forma Leucathea ${ }^{60}$ è

${ }^{49}$ Diodoro, V, 55, 7. Cf. Musti (1999), p. 68.

50 Novaro-LeFĖvre (2000), p. 47-52. Vd. pure SClafani (2007), p. 255.

51 Da una dedica a un sacerdote della dea, datata nel III sec. d.C. (IG XIV, 2433, 4). Cf. SAlviat (1992), p. 146-147; Hermary-TreZiny (2000), p. 148.

52 Aristotele, Retorica II, 23, 1400 b 5-6. Cf. CERRI (1994), p. 137 ss.

53 Cicerone, Discussioni Tuscolane I, 12, 28; Cicerone, Sulla natura degli dei III, 19, 48; Ovidio, Fasti VI 545; Plutarco, Vita di Camillo, 5, 1-2; Plutarco, Questioni romane, 267d-e; Iginio, Favole, 2, 224; Lattanzio, Istituzioni divine I, 21, 23; Servio, Commento a Virgilio, Eneide V 241; VII, 83; Servio, Commento a Virgilio, Georgiche I, 437. Cf. COARELLI (1988a), in part., p. 249-253, 328 ss., 338 ss.; (1988b), p. 144-145.

${ }^{54}$ Strabone, V, 2, 6, 226. Cf. ColonNA (1981), p. 13-37, in part., p. 29-34; (2000), p. 325-335.

55 Aristotele, Economico II, 2, 20, 1349b 33-35; Polieno, Stratagemmi V, 2, 21; Eliano, Storia varia I, 20.

${ }^{56} \mathrm{La}$ statua di culto, ripescata nelle acque del fiume Anio, teneva in mano il liber dei responsi della dea (Lattanzio, Istituzioni divine I, 6, 12). Cf. CHAMPEAux (1990b), p. 272, n. 3; (1990c), p. 825-826; (1997), p. 417, 419.

57 Servio, Commento a Virgilio, Eneide VII, 83-84. Cf. Cerri (1994), p. 142; A. Maggiani, ThesCRA III (2005), p. 70.

58 Pomponio Mela, II, 7, 121; Plinio, Storia naturale III 13, 83; Marziano Capella, 6, 644. Cf. Cerri (1994), p. 143 s., 146.

${ }^{59}$ Strabone, II 5, 20, 123; VI 1, 1, 252; VI 1, 6, 258; Plinio, Storia naturale III 13, 85. Cf. CERRI (1994), p. 146-149; MusTi (1999), p. 33-46.

${ }^{60}$ In un frammento di Conone il mito di Ino/Leucothea è legato alla fondazione del santuario oracolare dei Branchidi vicino Mileto: Conone, 26 F 1, 33 (ed. JACOBY). Vd. Polito (2006), p. 353-354. 
strettamente collegata col «retroterra metropolitano che è tessalo-beotico ed euboico insieme» ${ }^{61}$. La forma più antica del teonimo, attestata in Tessaglia (a Larisa, Pherai e Atrax $)^{62}$, si conserva anche nei nomi di feste o mesi, $\Lambda \varepsilon u x^{\prime} \theta \varepsilon \alpha$, $\Lambda \varepsilon u \varkappa \alpha \theta \varepsilon \omega \dot{\omega} \nu, \Lambda \varepsilon u \varkappa \alpha \theta \iota \omega ́ v$ dell'isola di Chio ${ }^{63}$ e di alcune città della costa anatolica ${ }^{64}$. La connessione del culto di Leucothea con le navigazioni focee e rodie nel Tirreno, proposta da altri studiosi, si fonda sulle attestazioni di Massalia, Elea e Lampsaco, altra colonia focea, oltre che sulla tradizione diodorea già richiamata ${ }^{65}$.

\section{Funzione delle sferette}

Vista la somiglianza fra le tre sferette, Marina Scafani ha avanzato l'ipotesi che provenissero tutte dall'unico contesto di rinvenimento noto, l'ambiente 2 della stoa ovest $^{66}$. Quest'ipotesi, a giudizio degli archeologi che hanno lavorato a Himera, deve essere esclusa per il fatto che lo scavo della stoa ovest era stato completato al tempo del ritrovamento delle sferette con i nomi di Eracle e Leucathea.

Quanto alle modalità d'impiego di questi piccoli oggetti, la Sclafani ha escluso sia che si tratti di ghiande missili - le ghiande missili hanno solitamente forma allungata - sia che si tratti di offerte votive - le offerte votive sono provviste in genere di una base che ne rende agevole l'esposizione su una superficie piana ${ }^{67}$. Esaminando la documentazione relativa ai culti di Zeus Soter ed Eracle, la studiosa ha sottolineato il carattere salvifico di queste divinità e si è soffermata poi sul culto di Leucothea, richiamando l'attenzione sulla sua diffusione nell'area tirrenica e sulle corrispondenze tra l'orizzonte cultuale imerese e quello di molte città, non solo greche, che si affacciano allo stesso mare: il culto di Zeus, Eracle e Lencathea a Himera rimanderebbe a quello di Tina, Hercle e Thesan nel santuario di

${ }^{61}$ Giangiulio (1986), p. 102-116. Vd. pure Miranda (1995), p. 30; EAD. (1998), p. 231 ss.

62 Larisa: Arvanitopoullos (1910), p. 378-382, n. 25; Fere: IG IX 2, 422, 2; SGDI I, 337; Atrax: SEG 35, 501.

63 SEG 17, 385, 25; SEG 22, 508A I; SEG 35, 501.

64 A Teo sono noti la festa dei $\Lambda \varepsilon u x^{\prime} \theta \varepsilon \alpha$ e il mese $\Lambda \varepsilon u x \alpha \theta \varepsilon \dot{\omega} v$ : SEG 2, 579-580; HerRmanN (1965), p. 37-38, 1l. 21, 38; ŠAHIN (1985), p. 13-16. Il mese $\Lambda \varepsilon u x \alpha \theta \varepsilon \omega ́ v$ ricorre pure a Eritre: EngelmanN, MerkelbaCh (1973), II, n. 201 C, 13-14; n. 391, e Magnesia: Kern (1900), n. 89, 6; n. 215, 71. Nella colonia focea di Lampsaco ricorre il mese $\Lambda \varepsilon u x \alpha \theta \imath \omega \dot{v} v$ FriSCH (1978), n. 9, 17. Resta incerta l'integrazione del mese $\Lambda$ sux' $\theta \varepsilon o \varsigma$ in un'epigrafe di Astypalaea: IG XII 3, 174; BLÜMEL (1992), I 34, 1.

${ }^{65}$ Cf. Peterson (1919), p. 200-202; Salviat (1992), p. 146-147; Cerri (1994), p. 144.

66 Allegro (1991), p. 65-72.

${ }^{67}$ A questo proposito non posso fare a meno di osservare che, visto che tra le offerte consacrate nei santuari greci si trovano i più svariati oggetti usati della vita quotidiana, dovremmo chiederci piuttosto quali fossero le funzioni per cui le sferette fossero adoperate prima della dedica. 
Pyrgi; l'associazione di Atena ed Eracle con le acque calde a Himera sarebbe confrontabile con quella di Menerva ed Hercle presso le Aquae Caeretanae ${ }^{68}$.

La trama delle affinità cultuali tra le città greche e non greche delle coste del Tirreno si infittisce se si considera che, accettando l'identificazione di 'E $\pi \iota \nu \nu \sigma \alpha-$ $\mu \varepsilon ́ v \alpha$ con Ilitia, nel contesto cultuale imerese troveremmo associate due divinità, che, per affinità o complementarità di caratteri e funzioni, sono state identificate, come abbiamo ricordato, sia con la dea di Pyrgi sia con la Mater Matuta del santuario romano di Sant'Omobono.

Fig. 3

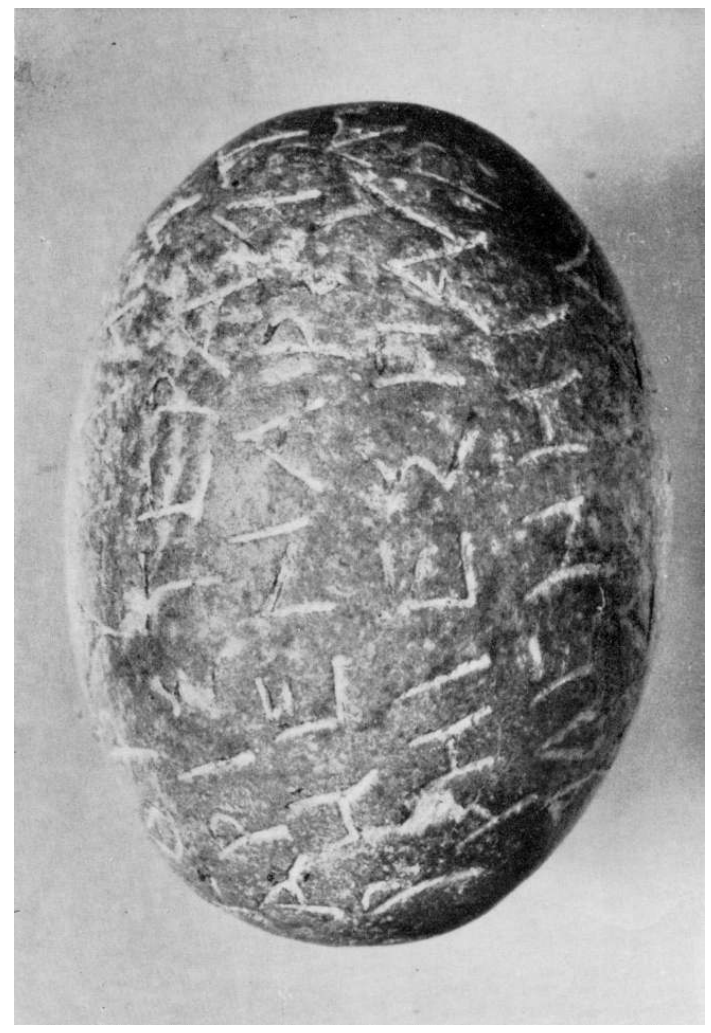

Proprio la proiezione tirrenica della colonia siceliota ${ }^{69}$ ha suggerito alla Sclafani l'ipotesi che le sferette iscritte fossero usate nelle operazioni di sorteggio richieste dalla cleromanzia, una pratica divinatoria che, anche se non estranea al mondo greco $^{70}$, era molto diffusa nell'ambito etruscoitalico. L'ipotesi si fonderebbe sulla "particolarissima e curata forma delle sfere», sul «carattere religioso dei nomi iscritti», sulla «provenienza» e infine «sulle analogie rilevabili con reperti etrusco-meridionali la cui interpretazione quali sortes è sicura» ${ }^{71}$.

A proposito della forma vorrei far notare che le sferette imeresi, pur adattandosi bene alle modalità di consultazione proprie della cleromanzia - potevano essere estratte da un contenitore, lanciate o fatte rotolare su una superficie piana -, non sono confrontabili tipologicamente, come rilevato peraltro dalla stessa Sclafani ${ }^{72}$, con nessuno degli oggetti

68 SCLAFANI (2007), p. 250-258.

${ }^{69}$ SCLAFANi (2007), p. 248-249.

70 Sulla cleromanzia nel mondo greco, vd. FONTENROSE (1978), p. 219-223, 228-229, 237 238, 367; GrotTANELLI (2001), p. 155-196; F. Graf, ThesCRA III (2005), p. 37-39.

71 SCLAFANI (2007), p. 259.

72 SCLAFANI (2007), p. 258. 
interpretati comunemente come sortes oracolari, noti dalle fonti letterarie e dai monumenti figurati (bassorilievi, specchi, vasi decorati a rilievo), o restituiti dai contesti archeologici (ciottoli con iscrizioni a rilievo, tavolette, dischetti, barrette di bronzo o piombo, astragali); tali oggetti provengono prevalentemente dall'area etrusco-laziale, ma sono attestati anche nell'Italia settentrionale, nel Sannio e in alcune città greche dell'Italia meridionale e della Sicilia ${ }^{73}$.

Tra i ciottoli con raffigurazioni ed epigrafi a rilievo, interpretati come «sortes» oracolari, ve ne sono tre con testi in greco, due di provenienza siciliana e uno di provenienza magno-greca: nell'esemplare che si dice rinvenuto a Camarina, figura una formula tipica delle dediche votive, costituita da un antroponimo al

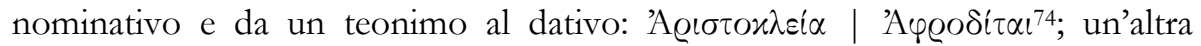
formula non distinguibile da quelle usate nelle dediche votive figura

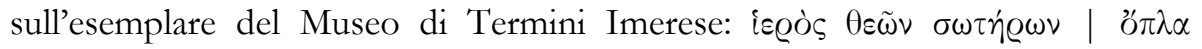
$\theta \varepsilon \tilde{\omega} \nu^{75}$; il ciottolo rinvenuto a Paestum sembra avere carattere funerario piuttosto

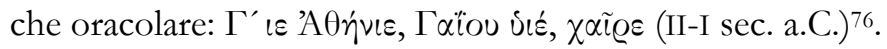

Si possono interpretare invece come responsi oracolari il testo del dischetto

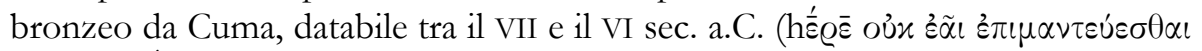

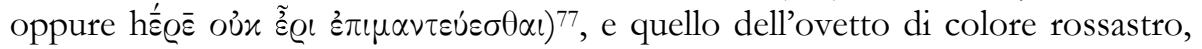
databile nel III sec. a.C., rinvenuto nei pressi di Siracusa: $x \circ \varrho u ́ \delta \alpha \lambda \lambda \alpha \mid \dot{\alpha} \varrho \iota \tau \varepsilon \varrho \alpha$

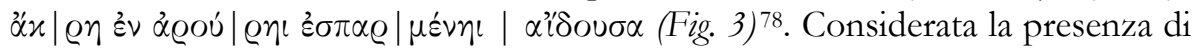
ionismi, Margherita Guarducci ha ipotizzato che quest'oggetto fosse stato importato dall'area delle colonie calcidesi o scritto nella dorica Siracusa da un abitante che parlava il dialetto ionico, ma non ha escluso la possibilità che fosse

73 Sulla cleromanzia nel mondo etrusco-italico, vd. CHAMPEAUX (1990a), p. 103-111; (1990b), p. 271-302; (1990c), p. 801-828; (1997), p. 405-438; BAGNASCO GIANNI (2001), p. 197-219; MAgGiani (1994), p. 68-78; (2005), p. 66-69, 73, 75-78.

74 Manganaro (1977), p. 150, tav. XLIII, 4; Maggiani (1994), p. 69, fig. 5; Sclafani (2007), p. 263-264, fig. 9. Secondo MAgGiani (1994, p. 69) Aristokleia avrebbe dedicato il ciottolo ad Afrodite «per grazia ricevuta e probabilmente per aver ottenuto un vantaggioso responso da parte della dea con la tecnica della litobolia».

75 Il ciottolo da Termini Imerese (SCLAFANi [2007], p. 262-264, fig. 5-8) non è compreso tra i sei esemplari con iscrizioni in greco, latico, osco ed etrusco elencati da MAGGIANI (1994, p. 6869). Potrebbe avere carattere votivo anche il ciottolo in calcare da Agrigento con figure a rilievo (facciata di un tempio ionico tetrastilo, tralcio d'edera e stella a otto punte) e firma dell'artista,

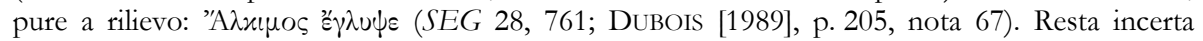
l'interpretazione del ciottolo proveniente da Selinunte, iscritto su due facce, pubblicato da G. RoCCA (2009, p. 269-273, n. 1).

76 MAGgIANI (1994), p. 68, 76, in part. nota 14, fig. 1, ha espresso dubbi sull'interpretazione funeraria dell'epigrafe.

77 Guarducci (1978), p. 81-82, fig. 29; Champeaux (1990b), p. 288-289, n. 36; DubOis (1995), p. 44-46, n. 14.

${ }_{78}$ Nella pianura del fiume Anapo, in un'area in cui venivano depositati gli scarichi di Siracusa. Museo Archeologico di Siracusa, Inv. 44016. GuARDuCCI (1959-1960), p. 275-278; MANNI PIRAINO (1963-64), p. 40-52; BE (1965), 501; 1966, 517. Manni Piraino, considerato il peso specifico, ritiene che l'ovetto debba essere di terracotta e non di pietra calcarea, come sostenuto da Guarducci. 
stato trasportato in Sicilia da una regione lontana. La studiosa, in un primo momento, ha interpretato il testo come «un augurio di felicità», facendo notare che la parte sinistra, 'infausta' per i Greci, è invece fausta nella divinazione italica; in seguito, riprendendo in esame il piccolo oggetto lo ha interpretato come una «sors appartenente ad un santuario oracolare in cui si avesse qualche

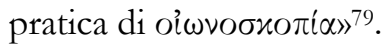

Visto che gli oggetti interpretati come sortes oracolari differiscono per la forma, il materiale e il contenuto delle iscrizioni, c'è da chiedersi se nel caso delle sferette di Himera i testi possano fornire qualche elemento a sostegno dell'ipotesi della connessione con una pratica cleromantica.

L'ipotesi della Sclafani, secondo cui il teonimo al genitivo o al nominativo «doveva indicare il dio (o gli dei) ai quali indirizzare le offerte» ${ }^{80}$, si fonda sul contenuto di molti responsi oracolari in cui figurano i nomi delle divinità alle quali il dio ordinava di sacrificare e offrire doni, ma l'esame delle varie forme di divinazione mediante sorteggio potrebbe suggerire una diversa spiegazione.

Pausania riferisce che, scendendo dalla città di Boura (Acaia) verso il mare si incontra una grotta - all'interno della quale si trova una statua di Eracle Bovgaïxós - dove è possibile ricevere oracoli per mezzo di un pinax e degli astragali. Chi volesse consultare il dio doveva pregare davanti alla statua, poi, avendo preso quattro astragali - tra quelli che si trovavano in gran numero vicino alla statua di Eracle - doveva lanciarli sulla $\tau \varrho^{\prime} \pi \varepsilon \zeta \alpha$ : le indicazioni scritte sul pinax avevano un'interpretazione appropriata per ciascuna combinazione di astragali ${ }^{81}$.

Altre informazioni importanti sulle procedure di consultazione degli oracoli mediante astragali provengono dalle collezioni più o meno frammentarie di responsi, datate nel II o tra il II e il III sec. d.C., attestate epigraficamente in una vasta area dell'Asia Minore sud-occidentale che si estende tra Frigia, Licia, Cilicia, Pisidia e Panfilia. Gli astragali hanno solo quattro facce su cui possono poggiare quando sono gettati su una superficie piana (le altre due facce sono convesse): a queste facce erano attribuiti i numeri 1, 3, 4, 6. Visto che le facce differiscono per forma e dimensioni, non era necessario segnare i numeri. Nelle epigrafi ciascun

\footnotetext{
${ }^{79}$ GUARDuCCI (1959-60), p. 276-277.
}

80 SCLAFANI (2007), p. 259.

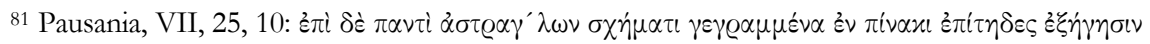
ध้ $\chi \varepsilon \iota ~ \tau o \tilde{~} \sigma \chi \eta \dot{n} \mu \alpha \tau o s$, «pour chaque configuration des osselets des indications qui figurent sur la tablette permettent d'interpréter de façon appropriée la configuration» (CASEVITZ, Paris, 2000). La mia interpretazione del passo si basa sul testo e sulla traduzione di Casevitz, per il quale «le mot schema a très vraisemblablement ici le sens de 'combinaison' et désigne la manière dont les osselets pouvaient combiner entre eux chacune de leurs quatre faces, sans qu'on puisse assurer qu'un chiffre y était gravé». Diversa la lezione di MogGi (Milano, 2000): «su ogni astragalo sono rappresentate delle figure e ciascuna figura è convenientemente spiegata sulla tavola». Alla consultazione dell'oracolo

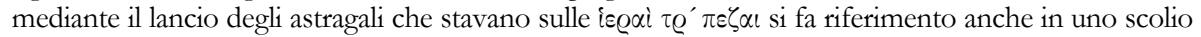
a Pindaro (Schol. vetera in Pind. Pyth. IV, 337-338b). Sull'oracolo di Boura, of. AmANDRY (1984), p. 377-378; OSANNA (1996), p. 240-242; GrotTANELLi (2001), p. 160-161. 
responso è preceduto da 5 o 7 numeri corrispondenti alle combinazioni dei lanci di 5 o 7 astragali (oppure a 5 o 7 lanci di un singolo astragalo), dalla rispettiva somma e infine da un teonimo al genitivo ${ }^{82}$. I responsi non sono individuati mediante il totale, ma mediante le combinazioni dei numeri (lo stesso totale potendo risultare da combinazioni diverse); ciascuna combinazione è contraddistinta mediante un teonimo al genitivo; se il nome di una divinità compare più volte è accompagnato generalmente da epiteti diversi ${ }^{83}$. Non è questa la sede per discutere se il teonimo fosse quello del dio o della dea da cui proveniva il testo oracolare o che guidava la mano che effettuava il lancio ${ }^{84}$, se ci fosse corrispondenza tra il responso e le prerogative della divinità a cui era intestato ${ }^{85}$.

La questione se le sferette bronzee da Himera fossero usate in qualche pratica divinatoria di carattere cleromantico, stante la mancanza di riscontri nei dati offerti dalla ricerca archeologica, è destinata almeno per il momento a restare aperta.

Le collezioni di oracoli per astragali, seppur molto lontane nel tempo e nello spazio, forniscono ad ogni modo un dato interessante per il tema in discussione: se il teonimo rendeva riconoscibile la combinazione di astragali e insieme il responso ad essa collegato, si può pensare che potesse esistere una procedura cleromantica nella quale l'estrazione di piccoli oggetti su cui erano incisi nomi divini, bastasse a individuare il responso corrispondente in una raccolta - che non doveva essere incisa necessariamente su pietra - a disposizione dei fedeli o del personale della sede dell'oracolo. Questa procedura è confrontabile con quella che doveva essere adottata negli oracoli alfabetici, attestati da alcune epigrafi rinvenute in una vasta area dell'Asia Minore, in larga misura sovrapponibile a quella da cui provengono gli oracoli per astragali ${ }^{86}$. Il fedele che andava a consultare uno di questi oracoli, estraendo da un contenitore un dado, una sferetta, un piccolo disco su cui figurava una lettera, poteva conoscere immedia-

\footnotetext{
82 GuARDuCCI (1978), p. 105-109; FonTENROSE (1978), p. 219-223, 228 s., 237 s., 367; ZEVI (1982), p. 605-609; F. Graf, ThesCRA III(2005), p. 37-39; Nollé (2007), p. 7-222.

83 Sugli astragali figurano talvolta nomi di dei o di eroi, quasi sempre in nominativo; questi nomi, sulla base del fatto che le fonti letterarie ricordano nomi di dei e di eroi usati per indicare alcune possibili combinazioni di numeri, sono stati interpretati come «marques» piuttosto che come «pièces du jeu». Non esiste però alcuna corrispondenza tra i nomi incisi sugli astragali e i nomi dei lanci tramandati letterariamente (AMANDRY [1984], p. 370-375, in part. p. 375). L'unica eccezione è costituita dal nome di Eracle che, oltre a comparire su un esemplare dall'antro Coricio, viene citato da Plutarco per indicare il lancio effettuato da un custode del tempio del dio che, essendo solito trascorrere il tempo libero giocando con i dadi o gli astragali, in assenza dei suoi abituali compagni di gioco, propose a Eracle una partita e, fissata la posta in gioco in caso di vittoria dell'uno o dell'altro giocatore, lanciò i dadi a nome suo e a nome del dio, assicurando la vittoria a quest'ultimo (Plutarco, Vita di Romolo, 5, 1; Questioni romane, 35, 272f).

84 GRAF (2005), p. 66.

85 Cf. NAOUR (1980), p. 34.

86 Guarducci (1978), p. 100-105; NAOur (1980), p. 22-36; RitTI (1989), p. 245-286; F. Graf, ThesCRA III (2005), p. 38-39; Nollé (2007), p. 223-280.
} 
tamente la risposta del dio andando a leggere il verso che iniziava con la stessa lettera sull'epigrafe.

Quanto all'esistenza nel mondo antico di collezioni di oracoli registrate su materiale deperibile, a disposizione di privati o di personaggi con responsabilità politiche o religiose ${ }^{87}$, oltre ai più famosi oracoli sibillini, mi piace ricordare $i$

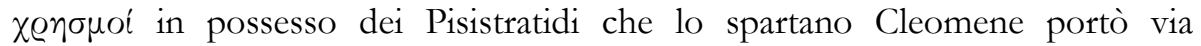

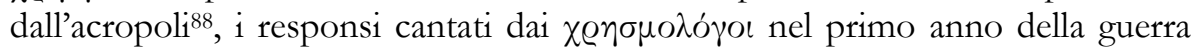
del Peloponneso, quando l'esercito spartano al comando di Archidamo, invasa l'Attica, giunse a sessanta stadi dalla città di Atene ${ }^{89}$, e infine gli oracoli di Bacide ricordati da Aristofane in una scena degli Uccelli dove tra i personaggi che sfilano di fronte a Pistetero, chiedendo di partecipare alla fondazione di

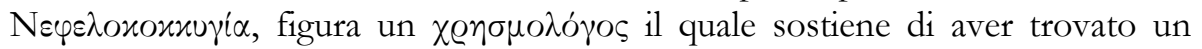

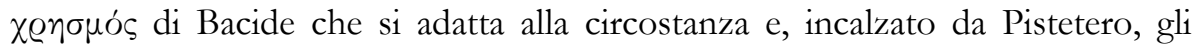
porge il papiro ( $\beta$ « $\beta$ iov) che lo contiene; a sua volta Pistetero, trovandosi a corto di argomenti, per confutare le affermazioni del suo interlocutore non riesce ad escogitare niente di meglio che leggere da un $\beta \iota \beta \lambda i o v$ un oracolo di Apollo che egli stesso aveva trascritto ${ }^{90}$.

Facoltà di Lettere - Università di Palermo

Antonietta BRUGNONE

Viale delle Scienze, Edificio 12

I - 90128 PALERMO

E-mail: antonietta.brugnone@alice.it

\section{Elenco delle illustrazioni}

Fig. 1: Sferetta bronzea iscritta da Himera.

Fig. 2: Sferetta bronzea iscritta da Himera.

Fig. 3: Uovo fittile iscritto da Siracusa.

${ }^{87}$ Sulle collezioni di oracoli, vd. FOnTENTOSE (1978), p. 145-165; W. BuRKERT, ThesCRA III (2005), p. 39-45; HENRICHS (2003), p. 216-222; BREMMER (2010), p. 14-16.

${ }^{88}$ Erodoto, V, 90, 2 - 91, 1; VII 6.

89 Tucidide, II, 21, 3.

${ }^{90}$ Aristofane, Uccelli, 959-991. 


\section{Bibliografia}

N. Allegro, «Il santuario di Athena sul Piano di Imera», in Di Terra in terra. Nuove scoperte archeologiche nella provincia di Palermo, Palermo, 1991, p. 65-72.

P. Amandry, «Os et coquilles», in L'antre Corycien, II, Athènes-Paris, 1984 (BCH, Suppl. IX), p. 347-380.

A.S. ARvanitopoullos, «Thessalikaì Epigraphaì, Eph. Arch. (1910), p. 378-382.

G. BAgnasco GiAnNi, «Le sortes etrusche», in F. Cordano, C. GrotTANELli (a cura di), Sorteggio pubblico e cleromanzia dall'antichità all'età moderna. Atti della Tavola Rotonda, Milano 26-27 gennaio 2000, Milano, 2001, p. 197-220.

F. BeChtel, Die Historischen Personennamen des Griechischen bis zur Kaiserzeit, Halle, 1917.

W. BLÜMEL, Die Inschriften von Knidos, Bonn, 1992.

N. BONACASA, «Noterelle di topografia storica sul temenos arcaico di Himera», Architettura e urbanistica nella Sicilia greca arcaica, CdA 19 (1980) [1994], p. 41-49.

—, "Il temenos di Himera», Secondo Quaderno Imerese. Studi e Materiali dell'Isituto di Archeologia dell" Università di Palermo, 3, Roma, 1982, p. 47-60.

—, «Himera. La saga di Eracle tra mito e storia», in Studi di filologia classica in onore di G. Monaco, Palermo, 1991, p. 1431-1439.

—, «Da Agrigento a Himera: la proiezione culturale», in L. BRACCESI, E. DE Miro (a cura di), Agrigento e la Sicilia greca. Atti della settimana di studio, Agrigento 2-8 maggio 1988, Roma 1992, p. $133-150$.

—, «Donari fittili nel temenos di Athena a Himera?», in R. GIGLI (a cura di), MEГA AAI NH $\Sigma$ OI. Studi dedicati a Giovanni Rizza per il suo ottantesimo compleanno II, 2005, p. 241-253.

L. Breglia Pulci Doria, «Atena e il mare: problemi e ipotesi sull'Athenaion di Punta della Campanella», in I culti della Campania antica. Atti del Convegno Internazionale di Studi in ricordo di Nazarena Valenza Mele, Napoli, 15-17 maggio 1995, Roma, 1998, p. 97-108.

J.N. Bremmer, «Manteis, Magic, Mysteries and Mythography: Messy Margins of Polis Religion?», Kernos 23 (2010), p. 13-35.

A. BRugnONE, «Gli alfabeti arcaici delle poleis siceliote e l'introduzione dell'alfabeto milesio», ASNP s. III, 25 (1995), p. 1297-1327.

M. Caccamo Caltabiano, s.v. Himera, LimC V (1990), p. 424-425.

L. CERCHIAI, «Sesso e classi di età nelle necropoli greche di Locri Epizefirii», in G. GNOLI, J.-P. VERNANT, La mort, les morts dans les societés anciennes, Paris, 1982, p. 289-298.

G. CERRI, «Elea, Senofane e Leucothea», in Forme di religiosità e tradizioni sapienziali in Magna Grecia. Atti del convegno, Napoli 14-15 dicembre 1993. AION, sez. filol.-lett., 16 (1994), p. 137-155.

J. Champeaux, «Les oracles de l'Italie antique : hellénisme et italicité», Kernos 3 (1990), p. 103111.

—, «Sors oraculi. Les oracles en Italie sous la République et l'Empire», MEFRA 102 (1990), p. 271 302.

—, «Sorts et divination inspirée. Pour une préhistoire des oracles italiques», MEFRA 102 (1990), p. 801-828.

—, «De la parole à l'écriture. Essai sur le langage des oracles», in J.-G. HeINTZ (a cura di), Oracles et prophéties dans l'antiquité. Actes du Colloque de Strasbourg, 15-17 juin 1995, Paris, 1997, p. 405438.

P. Chantraine, Dictionnaire étymologique de la langue grecque, Paris, 1968.

F. COARELLI, Il Foro Boario dalle origini alla fine della repubblica, Roma, 1988.

—, «I santuari, il fiume, gli empori», in Storia di Roma, 1. Roma in Italia, Torino, 1988, p. 127-151.

G. ColonNA, «La dea di Pyrgi: bilancio aggiornato dei dati archeologici (1978)», in Die Göttin von Pyrgi. Archaeologische, linguistische und religionsgeschicbtliche Aspekte. Akten des Kolloqiums, Tübingen, 16-17 Januar 1979, Firenze, 1981, p. 13-34. 
—, «ll santuario di Pyrgi dalle origini miti-storiche agli altorilievi frontonali dei Sette e di Leucotea», in Dèi ed eroi greci in Etruria. Atti del Colloquio internazionale, Roma, 1997, Scienze dell'Antichità 10 (2000), p. 251-336.

V. CONSOLI, «Il cosiddetto tempio della Vittoria a Himera. Per un'alternativa storico-religiosa», Workshop di Archeologia classica 5 (2008), p. 43-75.

E. De Miro, Agrigento II. 1. Santuari extraurbani. L'Asklepieion, Roma, 2003.

L. Deubner, Attische Feste, Berlin, 1932.

L. DuBOIS, Inscriptions grecques dialectales de Sicile. Contribution à l'étude du vocabulaire grec colonial, Rome, 1989 (Collection de l'École Francaise de Rome, 119).

-, Inscriptions grecques dialectales de Grande Grèce I. Colonies eubéennes. Colonies ioniennes. Emporia, Genève, 1995.

—, Inscriptions grecques dialectales de Sicile II, Genève, 2008.

H. Engelmann, R. MerkelBach, Die Inschriften von Erythrai und Klazomenai II, Bonn, 1973.

J. Fontenrose, The Delphic Oracle. Its responses and operations with a catalogue of Responses, Berkeley / Los Angeles / London, 1978.

P.R. Franke, M. Hirmer, Die griechische Münze, München, 1972.

P. FRISCH, Die Inschriften von Lampsakos, Bonn, 1978.

G. GÉRARD-ROUSSEAU, Les mentions religieuses dans les tablettes mycéniennes, Rome, 1968.

M. Giangiulio, «Per la storia dei culti di Crotone antica. Il santuario di Hera Lacinia. Strutture e funzioni cultuali, origini storiche e mitiche», Arcivio Storico Calabria e Lucania 49 (1982), p. 569.

—, «I culti», in Neapolis. Atti del XXV Convegni di studi sulla Magna Grecia, Taranto, 3-5 ottobre 1985, Napoli, 1986, p. 99-154.

—, Ricerche su Crotone arcaica, Pisa, 1989 (Scuola Normale Superiore di Pisa. Pubblicazioni della Classe di Lettere e Filosofia, 6).

F. GRAF, «Rolling the dice for an answer», in S.I. JoHnSTON, P.T. STUCK (a cura di), Mantikê. Studies in Ancient Divination, Leiden / Boston, 2005, p. 51-97.

C. Grotta, «Le iscrizioni greche», in M.L. Fama (a cura di), Il Museo Regionale "A. Pepoli” di Trapani. Le collerioni archeologiche, Bari, 2009, p. 375-384.

C. Grottanelli, «La cléromancie ancienne et le dieu Hermès», in F. Cordano, C. GrottaNELLi (a cura di), Sorteggio pubblico e cleromanzia dall'antichità all'età moderna. Atti della Tavola Rotonda, Milano 26-27 gennaio 2000, Milano, 2001, p. 155-196.

M. GuARDUCCI, «Nuove note di epigrafia siceliota arcaica», ASAA 37-38, n.s. 21-22 (1959-1960), p. 249-278.

—, Epigrafia greca, IV, Roma, 1978.

F. Gutmann, W. SChWABACHER, «Die Tetradrachmen und Didrachmenprägung von Himera (472-400 v. Chr.)», MBNG 47 (1929), p. 101-144.

A. Henrichs, «Hieroi Logoi and Hierai Bibloi: the (Un)written Margins of the Sacred in Ancient Greece», HSCP 101 (2003), p. 207-266.

P. Herrmann, «Antiochos der Grosse und Teos», Anadolu 9 (1965), p. 29-160.

A. Hermary, H. Tréziny, «Les cultes massaliètes: documentation épigraphique et onomastique», in Les cultes des cités phocéennes. Actes du colloque international organisé par le Centre Camille-Jullian, Aix-en-Provence/Marseille 1999, Aix-en-Provence, 2000, p. 147-157.

A. HeubeCK, «Etymologische Vermutungen zu Eleusis und Eileithyia», Kadmos 11 (1972), p. 8795.

O. KERN, Inscbriften von Magnesia am Meander, Berlin, 1900.

K. LatTe, Hesychii Alexandrini Lexicon I-II, Hauniae, 1966.

E. Lippolis, M. NAFissi, S. GarrafFo, Culti greci in Occidente I, Taranto, 1995. 
G. MADDOLI, «Manomissioni sacre in Eraclea Lucana (S.E.G. XXX, 1162-1170)», PP 41 (1986), p. 99-107.

—, «Asilo sacro, via di libertà. Per la storia di Eleutheria nella Grecia antica», AnnPerugia 25 (198788), p. 141-156.

A. MAgGiAnI, «Mantica oracolare in Etruria: litobolia e sortilegio», RdA 18 (1994), p. 68-78.

G. Manganaro, «Per la storia dei culti nella Sicilia greca», in Atti della I Riunione Scientifica della Scuola di perfezionamento in archeologia classica dell'Università di Catania, Siracusa, 24-27 novembre 1976, CrASA 16 (1977), p. 148-164.

—, «Istituzioni pubbliche e culti religiosi», in L. BracCeSI, E. De Miro (a cura di), Agrigento e la Sicilia greca. Atti della settimana di studio, Agrigento, 2-8 maggio 1988, Roma, 1992, p. 207-218.

—, «Sikelika, 1», QUCC 49 (1995), p. 93-109.

—, «Figurazioni e dediche religiose della Sicilia greca e romana», ZPE 113 (1996), p. 77-81.

-, «Mondo religioso greco e mondo "indigeno" in Sicilia», in C. AnTONETTi (a cura di), Il dinamismo della colonizzazione greca di età arcaica: metodologie e problemi a confronto, Venezia, 10-111995, Napoli, 1997, p. 71-82.

—, «Ancora sui culti della Sicilia greca: Zeus Soter e il fiume Sichas», SNR 82 (2003), p. 5-12.

M.T. Manni Piraino, «Intorno a un uovo fittile di allodola», Annali del Museo Pitrè 14-15 (196364), p. 40-52.

—, «Le iscrizioni», in Himera I. Campagne di scavo 1963-1965, Roma, 1970, p. 345-356.

—, «Alcune iscrizioni inedite dall'area sacra e dall'abitato di Himera», Kokalos 20 (1974), p. 265271.

—, «Intervento», Kokalos 30-31 (1984-85), p. 253-255.

L. Mildenberg-S. HurTer, The Arthur S. Dewing Collection of Greek Coins, New York, 1985.

E. Miranda, Iscrizioni greche d'Italia. Napoli. II, Roma, 1995.

—, «Sacerdozi a Napoli in età romana», in I culti della Campania antica. Atti del Convegno internazionale di Studi in ricordo di N. Valenza Mele, Napoli, $15-17$ maggio 1995, Roma, 1998, p. 231-238.

D. Musti, I Telchini, le Sirene. Immaginario mediterraneo e letteratura da Omero e Callimaco al romanticismo europeo, Pisa / Roma, 1999 (Quaderni della Rivista di Cultura classica e medievale, 1).

Ch. NAOUR, Tyriaion en Cabalide: épigraphie et géographie bistorique, Zutphen, 1980.

M.P. Nilsson, The Minoan-Mycenaean Religion and its Survival in Greek Religion, Lund, $1950^{2}$.

J. NoLLÉ, Kleinasiatische Losorakel. Astragal- und Alphabetchresmologien der hochkaiserzeitlichen Orakelrenaissance, Munchen, 2007 (Vestigia, 57).

D. Novaro-LefÈvre, «Le culte d'Héra à Pérachora (VIII s. - VI s.) : essai de bilan», REG 113 (2000), p. 42-69.

M. Osanna, Santuari e culti dell'Acaia antica, Napoli, 1996.

R. PARKER, «Demeter, Dionysios and the Spartan Pantheon», in Early Greek Cult Practice. Proceedings of the Fifth International Symposium at the Swedish Institute at Athens, $26-29$ june 1986, Stockholm, 1988 (ActaAth-4ㅇ 38), p. 99-104.

R.M. Peterson, The Cults of Campania, Rome, 1919 (Papers and Monographs of the American Academy in Rome, 1).

J. Puhvel, «Eleuthér and Oinoatis: Dionysiac Data from Mycenaean Greece», in Mycenaean Studies. Proceedings of the third International Colloquium for Mycenaean Studies, 4-8 september 1961, Madison, 1964, p. 161-170.

T. RitTI, «Oracoli alfabetici a Hierapolis di Frigia», XIV Miscellanea Greca e Romana, Roma, 1989, p. 245-286.

G. Rocca, «Due inediti da Selinunte», in C. AntonetTi, S. De Vido, Temi selinuntini, Pisa, 2009, p. 269-275.

S. ŠAHIN, «Ein neues Dekret der Symmoria zu Ehren ihrer Prostatai in Teos», EA 5 (1985), p. 13-17. 
F. SALVIAT, «Sur la religion de Marseille grecque», in M. BATS et al. (a cura di), Marseille grecque et la Gaule. Actes du Colloque international d'archéologie et d'histoire, Aix-en-Provence, 1992 (Études massaliètes, 3), p. 141-150

F. SARTORI, «Ancora sulle dediche a Demetra in Eraclea Lucana», in Mélanges P. Lévêque 6, Paris, 1992, p. 269-277.

M. SCLAFAnI, «Zeus Soter, Eracle, Leukathea e tre 〈sortes» dall'antica Himera», $M D A I(R) 113$ (2007), p. 247-265.

W. SCHUlZE, Quaestiones epicae, Gütersloh, 1892.

R. Spadea (a cura di), Il tesoro di Hera. Scoperte nel santuario di Hera Lacinia a Capo Colonna di Crotone, Milano, 1996.

M. TORELli, «I culti di Imera tra storia e archeologia», in G. FIorentinI et al. (a cura di), Archeologia del Mediterraneo. Studi in onore di Ernesto De Miro, Roma, 2003, p. 671-683.

A. Tusa Cutroni, «La monetazione di Himera: aspetti e problemi», in Quaderno Imerese. Studi e Materiali dell'Istituto di Archeologia dell'Università di Palermo 1, Roma, 1972, p. 111-122.

J. WaCKernageL, «Griechische Miszellen», Glotta 14 (1925), p. 36-67.

F. ZEvI, «Oracoli alfabetici, Praeneste e Cuma», in АПАРХАI. Nuove ricerche e studi sulla Magna Grecia e la Sicilia antica in onore di P.E. Arias II, 1982, p. 605-609. 